\title{
Editorial: Progress in surgical management of ocular trauma
}

The availability of new methods of treatment and the development of new instruments has had the effect of a fairly rapid evolution in the treatment of perforating injuries to the eye, leading to an obvious improvement in results.

No longer is the retention of a safe eye a satisfactory criterion of success; we must now aim also at a return to normal function.

The trends have been largely predictable. The most important has been the control of infection which in the past not only led to a rapid destruction of the eye but also precluded any primary surgery other than an immediate repair and closure of the wound often after the sacrifice of parts of the uvea and other intraocular contents.

It is difficult to imagine that it was sometimes only possible to cover a leaking corneal wound with a conjunctival flap because surgical facility was less available and suture materials were too coarse for accurate repair.

The introduction of the operating microscope into ophthalmology and the finer instruments and materials which followed led to a more accurate control of wound closure and easier reconstruction of the normal anatomy of the eye.

Re-formation of the anterior chamber with air helped to improve results but the re-formation of the chamber with a physiological solution reflected a more accurate wound closure-watertight instead of airtight.

Not long ago any iris which was prolapsed would be excised; a few surgeons were tempted to replace iris which appeared undamaged in a fresh perforation just as they would try to replace an iris prolapse which occurred after intraocular surgery. The result of this action was unexpectedly good; the eye with replaced iris was often quiet more quickly than a similar eye in which iris had been abcised. Not only that but the eye would have the advantage of a normally acting iris diaphragm and a more normal cosmetic appearance.

Repair of a torn iris diaphragm is being performed more widely and is likely to become the common practice when surgeons are convinced of the safety of iris suturing. The re-formation of the iris diaphragm helps to prevent anterior synechia of the iris to the wound or the angle.

Thus the last few years have seen the development of more complex reconstruction and more comprehensive primary surgery. Many eyes which would have been lost can now be seen to recover not only potential function but also a true return to binocular vision.

Further progress is to be expected in the management of traumatic cataract. It is possible to go further in primary surgery than simply removing swollen lens matter. Instruments with controlled infusion and aspiration coupled with cutting devices and ultrasound emulsification make it possible to remove the whole of the lens cortex with a chosen portion of the lens capsule. If damage involves the vitreous as well then a partial vitrectomy can prevent the rather serious complications of coexistent lens and vitreous damage. There is a suggestion that in cases of early infection and when inflammatory membranes are beginning to form, the radical approach can be extended to the removal of infective and inflammatory foci with improved results.

Some of the fields of advance are still controversial. The use of an intraocular lens to replace a traumatic cataract is advocated by some and condemned by others. There can be no doubt, however, that if a safe intraocular lens is available then its use in such cases permits a closer approach to normal function than by any other means.

These comments are related to the advances in the treatment of perforating wounds of the eye. The prognosis in blunt injury has also benefited from better appreciation of the pathology of vitreoretinal adhesions and the risks of retinal detachment. Progress in the treatment of burns has shown the need for early protection and deferred reconstruction.

Trauma is not the most prevalent cause of blindness in the world but it is certainly the commonest cause of unilateral loss of vision and it affects all countries. It has been justifiably included among the major projects in this Prevention of Blindness Year of the World Health Organization. 